\title{
Article \\ Inducible Enrichment of Osa-miR1432 Confers Rice Bacterial Blight Resistance through Suppressing OsCaML2
}

\author{
Yanfeng Jia, Quanlin Li, Yuying Li, Wenxue Zhai, Guanghuai Jiang * and Chunrong Li * \\ Institute of Genetics and Developmental Biology, Chinese Academy of Sciences, Beijing 100101, China; \\ yfjia@genetics.ac.cn (Y.J.); quanlinli@genetics.ac.cn (Q.L.); lyear@genetics.ac.cn (Y.L.); \\ wxzhai@genetics.ac.cn (W.Z.) \\ * Correspondence: ghjiang@genetics.ac.cn (G.J.); crli@genetics.ac.cn (C.L.)
}

check for updates

Citation: Jia, Y.; Li, Q.; Li, Y.; Zhai, W.; Jiang, G.; Li, C. Inducible Enrichment of Osa-miR1432 Confers Rice Bacterial Blight Resistance through Suppressing OsCaML2. Int. J Mol. Sci. 2021, 22, 11367. https:// doi.org/10.3390/ijms222111367

Academic Editor: Karen Skriver

Received: 25 August 2021

Accepted: 14 October 2021

Published: 21 October 202

Publisher's Note: MDPI stays neutral with regard to jurisdictional claims in published maps and institutional affiliations.

Copyright: (c) 2021 by the authors. Licensee MDPI, Basel, Switzerland. This article is an open access article distributed under the terms and conditions of the Creative Commons Attribution (CC BY) license (https:// creativecommons.org/licenses/by/ $4.0 /)$.

\begin{abstract}
MicroRNAs (miRNAs) handle immune response to pathogens by adjusting the function of target genes in plants. However, the experimentally documented miRNA/target modules implicated in the interplay between rice and Xanthomonas oryzae pv. oryzae (Xoo) are still in the early stages. Herein, the expression of osa-miR1432 was induced in resistant genotype IRBB5, but not susceptible genotype IR24, under Xoo strain PXO86 attack. Overexpressed osa-miR1432 heightened rice disease resistance to $\mathrm{Xoo}$, indicated by enhancive enrichment of defense marker genes, raised reactive oxygen species (ROS) levels, repressed bacterial growth and shortened leaf lesion length, whilst the disruptive accumulation of osa-miR1432 accelerated rice susceptibility to Xoo infection. Noticeably, OsCaML2 (LOC_Os03g59770) was experimentally confirmed as a target gene of osa-miR1432, and the overexpressing OsCaML2 transgenic plants exhibited compromised resistance to Xoo infestation. Our results indicate that osa-miR1432 and OsCaML2 were differently responsive to Xoo invasion at the transcriptional level and fine-tune rice resistance to Xoo infection, which may be referable in resistance gene discovery and valuable in the pursuit of improving Xoo resistance in rice breeding.
\end{abstract}

Keywords: rice bacterial blight; osa-miR1432; OsCaML2

\section{Introduction}

In plants, microRNAs (miRNAs) manipulate various biological processes, including developmental plasticity, stress responses and symbiotic/parasitic interactions by regulating target gene expression [1]. With plants predominantly dependent on resistance protein-coding genes to execute immunity against invading pathogen, miRNAs may function as crucial regulators in plant immune responses. The current knowledge of miRNA/target modules in plant-pathogen interaction are mainly concentrated on the pathosystems of plant and fungi or viruses, and only small-scale reports were involved in plant and bacteria. Therefore, to enrich the evidence of miRNA/target modules on plant bacterial disease would contribute to the overall mechanism dissection of plant-pathogen interaction and disease management, especially in crop resistance breeding.

Rice (Oryza sativa L.) offers calorie intake for more than half of the world's population, the worldwide production of which is restricted by the bacterial pathogen Xanthomonas oryzae pv. oryzae (Xoo) [2-4]. Multivarious conventional measures, including germicidal chemicals, biological agents and resistance breeding, are widely employed to control Xoo infection in rice cultivation. Unfortunately, the extensive use of germicidal chemicals brings environmental pollution and the tachytelic evolution of drug-resistant Xoo populations [5,6], and biological agents are not always effective. The deployment of cultivars carrying resistance gene, the most economical, environmentally friendly and effective method, is obstructed because of resistance loss and the emergence of new Xoo pathotypes [7-9]. Currently, an effective strategy for maintaining rice resistance to Xoo pathotypes is to excavate new resistance genes or reprogram the responsive patterns of TALE-triggered susceptibility genes, which is separately achieved by genome-wide association study 
(GWAS) approaches [10,11] and Cas9-mediated cutting events [12,13]. With the exception of the GWAS and TALE-dependent resistance gene mining methods, miRNA-targeted genes may provide another access to mine resistance-associated genes.

Although abundant candidate targets of miRNAs have been monitored via the bioinformatic crosstab survey of small RNA sequencing data and transcriptome or degradome data in Xoo-challenged rice leaves [14,15], empirical investigations on the functionality characterization of miRNA/target modules in rice-Xoo interaction are slowly releasing. Osa-miR1861k may render rice immune to Xoo infection by targeting LOC_Os03g18710 and LOC_Os06g44970 [16]. Conversely, osa-miR169o inhibits the accumulation of the NF-YA4 gene, which impedes $P R$ and NRT2 transcription and increases susceptibility to Xoo in rice [17]. The aforementioned targets of miRNAs were presented indirectly for rice defense against Xoo. Importantly, the transcript enrichment of OsIPA1, one target of osa-miR156, weakens GA-mediated Xoo susceptibility by stabilizing OsSLR1 [18], and the quantitative resistance of the OsNBS8R gene to Xoo was attenuated by non-TAL effector XopQ-inducible osa-miR1876 through DNA methylation [19], which were direct proofs of miRNA/target modules functioning in rice-Xoo interaction.

Pioneer reports uncovered that osa-miR1432 boosts the filling rate by targeting OsACOT [20] and may be responsive to Xoo stimulus in rice [15]. In this study, we found that osa-miR1432 is induced specifically by the Xoo strain PXO86 in rice-resistant genotype IRBB5 and positively regulates rice resistance against Xoo. Furthermore, we experimentally demonstrated that LOC_Os03g59770 (OsCaML2) is a genuine target of osa-miR1432, and OsCaML2 overexpressors develop disease susceptibility to Xoo.

\section{Results}

\subsection{Osa-miR1432 Expression Is Induced in Resistant Genotypes during Xoo Invasion}

In our previous small RNA sequencing data, osa-miR1432 was differentially responsive to Xoo strain PXO86 invasion in the susceptible genotype IR24 and resistant genotype IRBB5 [16]. To confirm this, reverse transcription quantitative PCR (RT-qPCR) assays were executed to depict the responsive pattern of osa-miR1432 at the early stage of Xoochallenged IR24 and IRBB5 (Figure 1A). In contrast to mock treatments, Xoo-responsive expression of osa-miR1432 showed no obvious difference in infected IR24 leaves except for 24 and $48 \mathrm{~h}$ post inoculation (hpi), with reduced accumulation. However, a higher enrichment of osa-miR1432 was observed from $8 \mathrm{hpi}$ to $96 \mathrm{hpi}$ in Xoo-treated IRBB5 than in mock-treated plants (Figure 1B), indicating that osa-miR1432 is specifically induced by Xoo strain PXO86 invasion in IRBB5. These results suggest that osa-miR1432 may engage in rice immunity against $X o o$ invasion.

\subsection{Overexpressing Osa-MiR1432 Imparts Rice Resistance to Xoo Infection}

To verify whether osa-miR1432 mediates disease resistance to Xoo infection in rice, overexpressing osa-miR1432 (OE1432) transgenic plants were generated under the Taipei 309 (TP309) background. Out of 32 independent transgenic lines, two lines with high expression levels of osa-miR1432 were chosen to evaluate the disease reaction (Figure 2A). The leaf disease lesions caused by Xoo strain PXO86 in transgenic plants OE1432-1 and OE1432-7 were shorter than those of the control TP309 (Figure 2B,C), implying that osamiR1432 may operate as a positive regulator in rice resistance to Xoo infection. Subsequently, the leaf lesions of OE1432 lines suffering the effects of Xoo strains PXO112, PXO99 and PXO339 were measured and weaker relative to TP309 (Figure S1A-F). These results suggest the universality of overexpressing osa-miR1432 in strengthening rice immunity to Xoo infection. 
A

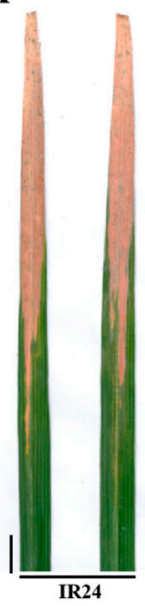

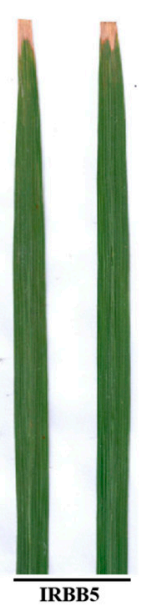

B

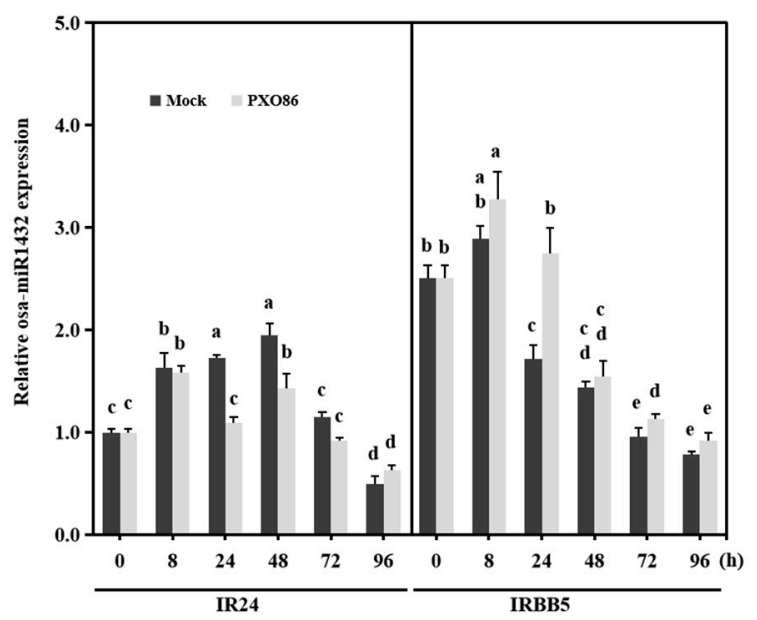

Figure 1. Differential expression patterns of osa-miR1432 in susceptible and resistant genotypes upon Xoo infection. (A) Phenotype of representative leaf sections from susceptible genotype IR24 and resistant genotype IRBB5 at 20 dpi with Xoo strain PXO86. (B) Accumulation of osa-miR1432 was monitored using RT-qPCR in susceptible and resistant genotypes under Xoo strain PXO86 and mock treatment. Scale bar, $2 \mathrm{~cm}$. Error bars indicate standard deviation $(n=3)$. Different letters above the bars indicate significant differences $(p<0.01)$ determined by one-way analysis of variance (ANOVA) followed by post hoc Tukey HSD analysis.
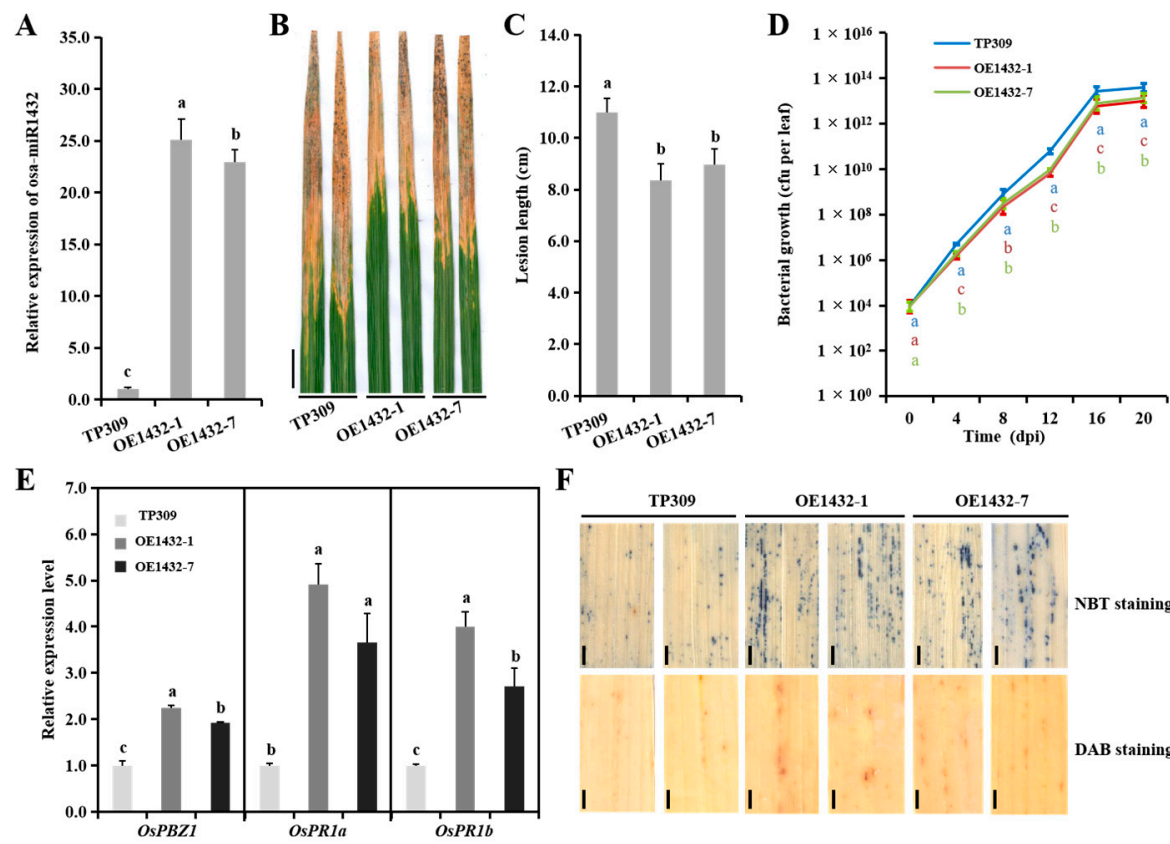

F

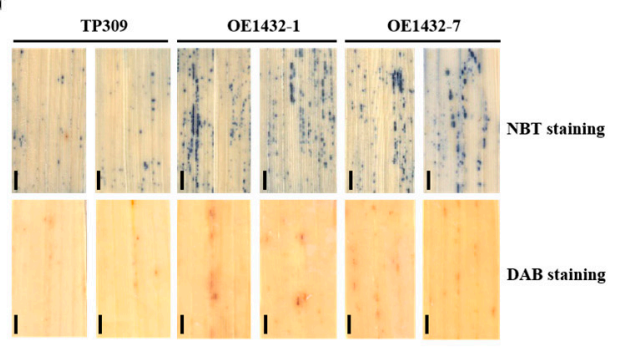

Figure 2. Osa-miR1432 modulates Xoo resistance in rice. (A) The expression detection of osa-miR1432 in representative overexpressing transgenic lines. SnRNA U6 served as the internal reference. (B) Lesions on OE1432 lines (OE1432-1 and OE1432-7) and the TP309 at 20 dpi. Scale bar, $2 \mathrm{~cm}$. (C) Lesion lengths on OE1432 plants $(n=20)$ at 20 dpi. (D) Bacterial growth indicated by the numbers of colony-forming units (cfu) per leaf in OE1432 lines and TP309. (E) The basic expression of OsPBZ1, OsPR1a and OsPR1b in OE1432 lines and TP309. (F) The histochemical detection of ROS levels using NBT and DAB staining in OE1432 lines and TP309 at 4 dpi. Error bars indicate standard deviation $(n=3)$. Scale bar, $2 \mathrm{~mm}$. Different letters above the bars indicate significant differences $(p<0.01)$ determined by one-way analysis of variance (ANOVA) followed by post hoc Tukey HSD analysis. 
In order to determine how osa-miR1432 blocks the pathogenicity of Xoo in rice, the typical immune response indicators were analyzed, including the initial expression of defense marker genes, the production of reactive oxygen species (ROS) and the bacterial growth curve. Firstly, the intrinsic expression levels of the defense marker genes, OsPBZ1 (Oryza sativa pathogenesis-related protein 10a) [21], OsPR1a (pathogenesis-related protein 1a) and OsPR1b (pathogenesis-related protein 1b) [22], in OE1432 plants were examined and superior to their counterparts in TP309 plants (Figure 2E), suggesting that OE1432 plants may possess a faster immune response. Secondly, $\mathrm{O}_{2}{ }^{-}$and $\mathrm{H}_{2} \mathrm{O}_{2}$, the main form of ROS, were found at higher levels at 4 days post inoculation (dpi) in OE1432 plants compared with TP309 plants (Figure 2F), suggesting that OE1432 plants may employ more severe cell damage to execute disease resistance. Finally, the growth rates of Xoo strain PXO86 were lower in OE1432 transgenic rice plants than those in TP309 plants, ranging from $4 \mathrm{dpi}$ to $20 \mathrm{dpi}$ (Figure 2D), implying that osa-miR1432-mediated resistance may act in the inhibition of Xoo strain multiplication. These data indicate that overexpressing osa-miR1432 reinforces rice resistance against Xoo strain PXO86 through the elevated expression of defense marker genes, ROS production and the decreased cell number of Xoo strain PXO86.

\subsection{Osa-MiR1432 Knockdown Plants Exhibit Aggravated Susceptibility to Xoo Infection}

To further notarize that osa-miR1432-mediated rice immunity depends on its inducible expression, we generated endogenous osa-miR1432 knockdown (MIM1432) lines using short tandem target mimic technology $[23,24]$ under the TP309 background. Two lines with notably reduced accumulation of osa-miR1432 were screened from 21 independent transgenic plants for disease assay (Figure 3A). By inoculating the Xoo strain PXO86, MIM1432-3 and MIM1432-8 lines presented more severe lesions, ranging from $13.0 \mathrm{~cm}$ to $13.3 \mathrm{~cm}$, than TP309, $11.0 \mathrm{~cm}$, in the infected leaves (Figure 3B,C). Consistent with the susceptible phenotype, the inherent transcripts of OsPBZ1, OsPR1a and OsPR $1 b$ were repressive in MIM1432 lines (Figure 3E). Similarly, responsive ROS levels for Xoo infection were low, at 4 dpi, in MIM1432 plants versus TP309 (Figure 3F). Bacterial growth survey results reflected that the PXO86 cells on the leaves of MIM1432 plants were one order of magnitude higher than those in TP309 plants after the middle stage of infection (Figure 3D). Additionally, the MIM1432 plants recapitulated the susceptibility under PXO112, PXO99 and PXO339 strain treatments (Figure S2A-F). These results demonstrate that interrupted osa-miR1432 weakens rice resistance against Xoo infection.

\subsection{Osa-miR1432 Targets the Transcripts of OsCaML2 in Plants}

Generally, disease resistance was conferred by the corresponding resistance gene in plant-pathogen interaction. To mine the downstream targets of osa-miR1432 in regulating rice immunity, eight potential targets for osa-miR1432 were predicted using the psRNATarget tool (http:/ / plantgrn.noble.org/psRNATarget/ accessed on 22 May 2020) with default parameters (Table S2). Coincidently, three potential targets, LOC_Os03g59770 (OsCaML2), LOC_Os04g08350 (OsCAS) and LOC_Os05g07210 (OsZIP6), also existed in high-quality IR24-D and IRBB5-D degradome data after removing adaptor sequences and low-quality reads [15], and then were further investigated in osa-miR1432 transgenic plants. As expected, the basic mRNA levels of all three targets were signally suppressive in OE1432 lines and cumulative in MIM1432 lines (Figure 4A). Simultaneously, all these genes were differentially expressed in IR24 and IRBB5 upon infection of Xoo strain PXO86 (Figure 4B-D). However, compared with the mock treatment, the Xoo-responsive patterns of OsCaML2 and OsCAS in IR24 and IRBB5 were, to some extent, opposite to osa-miR1432 in $3 \mathrm{dpi}$, whilst the expressing trend of OsZIP6 was in keeping with osa-miR1432 (Figure 4D). Noteworthily, from the perspective of Xoo-dependent expression, the opposite and similar transcriptional trend, ranging from $8 \mathrm{hpi}$ to $72 \mathrm{hpi}$ of OsCaML2 and OsCAS to osa-miR1432, appeared in Xoo-treated IRBB5, respectively. Thus, OsCaML2 was speculated as the best prospective target of osa-miR1432 in rice-Xoo interaction. 
To further affirm the regulatory relationship between OsCaML2 and osa-miR1432, a green fluorescent protein (GFP)-based reporter assay (Figure S3A) was conducted to transiently express OsCaML2-GFP or togetherwith osa-miR1432 in Nicotiana benthamiana. When OsCaML2-GFP and osa-miR1432 were co-expressed in Nicotiana benthamiana, the signal intensity of OsCaML2-GFP was gradually decreased with incremental proportion of osa-miR1432 in the injected mixture, accompanying no obvious change in GFP intensity of the control vector with or without osa-miR1432 under the same conditions (Figure S3B). To test whether MIM1432 could rescue the silencing of OsCaML2-GFP guided by osa-miR1432, MIM1432 was added stepwise to the infiltrated mixture of OsCaML2-GFP and osa-miR1432, and the GFP signals became increasingly stronger (Figure S3C). Taken together, the above results suggest that OsCaML2 is an authentic target of osa-miR1432 in rice.
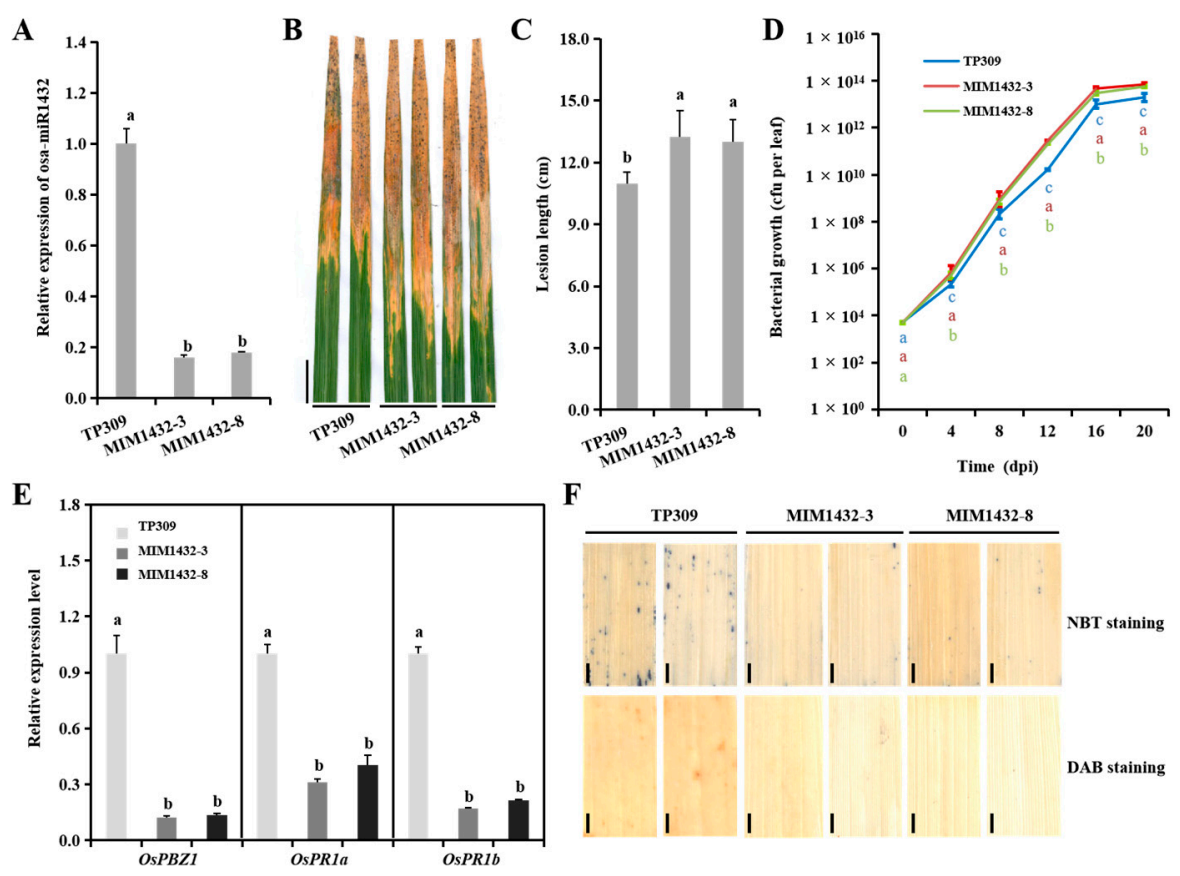

Figure 3. Restraining the expressing of osa-miR1432 results in susceptibility to Xoo attack in rice. (A) The expression detection of osa-miR1432 in MIM1432 lines, SnRNA U6, served as the internal reference. (B) Lesions on MIM1432 lines (MIM1432-3 and MIM1432-8) and TP309 at 20 dpi. Scale bar, $2 \mathrm{~cm}$. (C) Lesion lengths on MIM1432 lines $(n=20)$ at 20 dpi. (D) Bacterial growth as indicated by the numbers of colony-forming units (cfu) per leaf in MIM1432 lines and TP309. (E) The basic expression of OsPBZ1, OsPR1a and OsPR1b in MIM1432 transgenic lines and TP309. (F) The histochemical detection of ROS levels using NBT and DAB staining in MIM1432 lines and TP309 at 4 dpi. Error bars indicate standard deviation $(n=3)$. Scale bar, $2 \mathrm{~mm}$. Different letters above the bars indicate significant differences $(p<0.01)$ determined by one-way analysis of variance (ANOVA) followed by post hoc Tukey HSD analysis. 
A
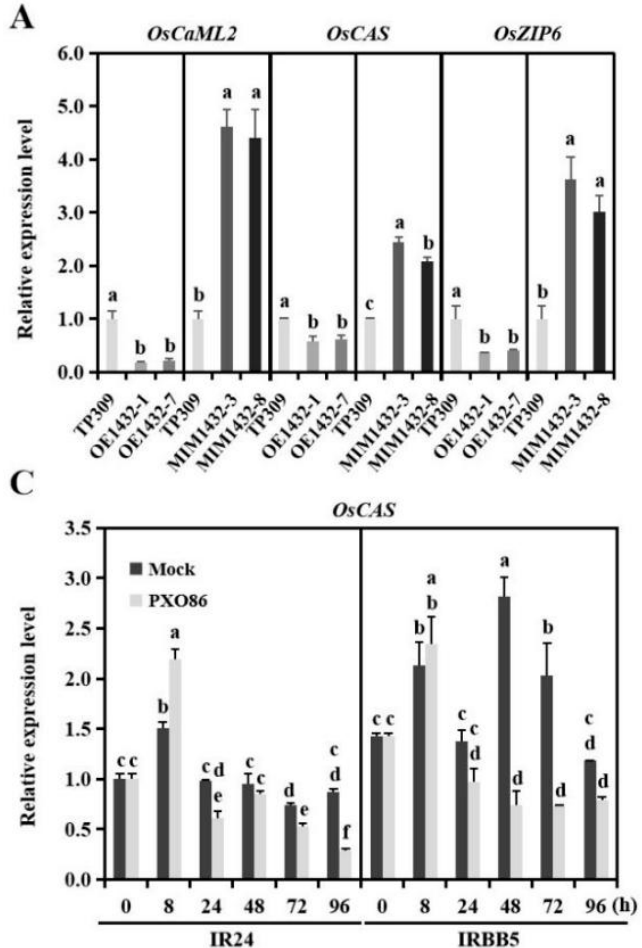

B

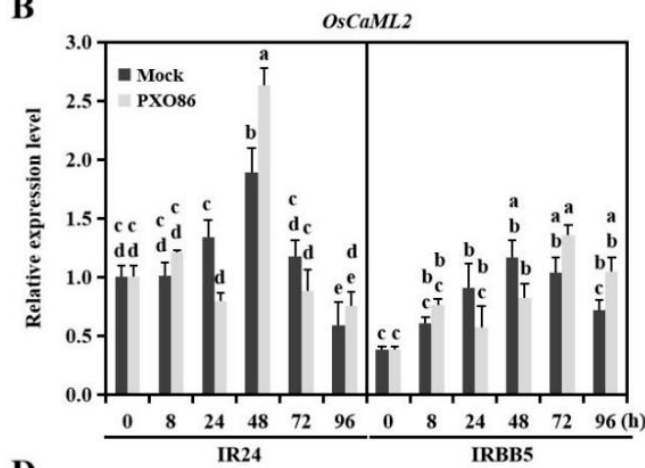

D

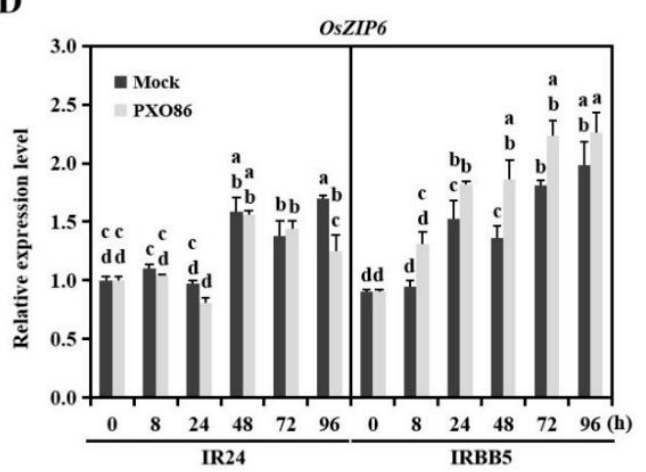

Figure 4. The candidate targets of osa-miR1432 have differential expression profiles in susceptible and resistant genotypes upon Xoo infection. (A) The relative expression levels of the candidate targets in the OE1432 and MIM1432 lines. (B-D) The expression pattern of OsCaML2, OsCAS and OsZIP6 in susceptible and resistant genotypes upon Xoo or mock treatment. Error bars indicate standard deviation $(n=3)$. Different letters above the bars indicate significant differences $(p<0.01)$ determined by one-way analysis of variance (ANOVA) followed by post hoc Tukey HSD analysis.

\subsection{Overexpression of OsCaML2 Phenocopies the Susceptibility of MIM1432 Plants}

To investigate the underlying biological function of OsCaML2 in rice, phylogenetic tree analysis and subcellular localization were implemented. Phylogenetic tree analysis uncovered that all 38 homologues of OsCaML2 were from plants, especially in Gramineae (Figure S4). Unfortunately, the relevant biological function studies of these 38 proteins have not been reported. Concurrently, the functional compartment localization exposed that OsCaML2 was expressed in both the nucleus and cytoplasm in Nicotiana benthamiana cells (Figure S5), meaning OsCaML2 may participate in the signal transduction of the nucleus and cytoplasm.

To experimentally ascertain the role of OsCaML2 in Xoo infection, OsCaML2overexpressing (OEOsCaML2) plants, driven by UBI promoter, were created, and the disease resistance of two highly expressed lines, OEOsCaML2-5 and OEOsCaML2-6, were examined by inoculating the Xoo strain PXO86. The inoculated leaves from OEOsCaML2-5 and OEOsCaML2-6 lines proved to have longer lesion lengths than those of TP309 plants (Figure 5A-C), and the disease symptoms were supported by the transcript levels of defense marker genes, the histochemical detection of ROS and bacterial growth number in OsCaML2 transgenic lines (Figure 5D-F). These results indicate that OsCaML2 may negatively regulate the immunity of rice to Xoo infection. 
A

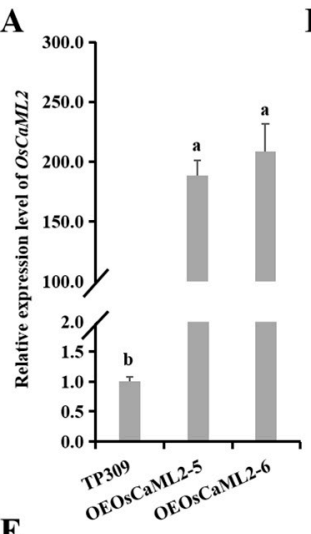

$\mathbf{E}$

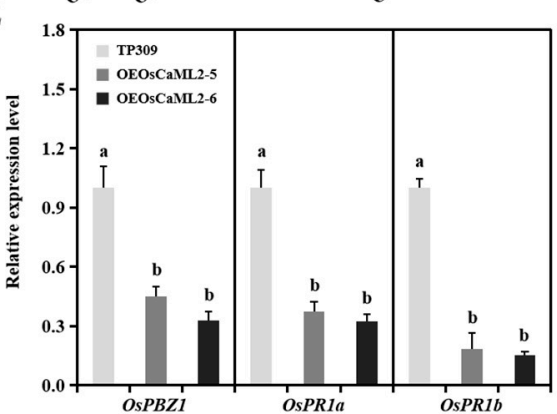

B

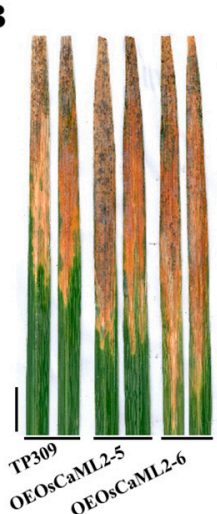

C

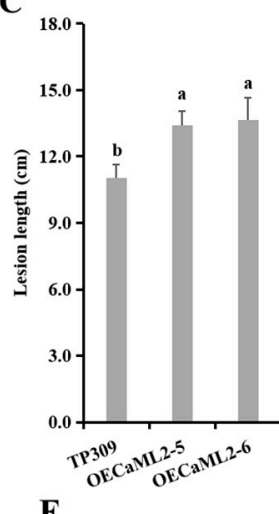

F

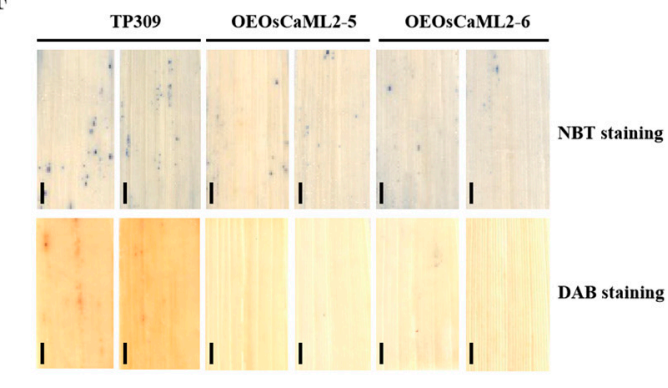

Figure 5. OsCaML2 negatively regulates disease resistance against Xoo in rice. (A) Accumulation of OsCaML2 in the OEOsCaML2 transgenic lines. (B) Lesions on respective OEOsCaML2 lines (OEOsCaML2-5 and OEOsCaML2-6) and the TP309 at 20 dpi. Scale bar, $2 \mathrm{~cm}$. (C) Lesion lengths on OEOsCaML2 plants $(n=20)$ at 20 dpi. (D) Bacterial growth as indicated by the numbers of colony-forming units (cfu) per leaf for OEOsCaML2 lines and the TP309. (E) Expression pattern of OsPBZ1, OsPR1a and OsPR1b in OEOsCaML2 transgenic lines and the TP309. (F) The histochemical detection of ROS levels using NBT and DAB staining in OEOsCaML2 transgenic lines and the TP309 after Xoo infection. Error bars indicate $\mathrm{SD}(n=3)$. Scale bar, $2 \mathrm{~mm}$. Different letters above the bars indicate significant differences $(p<0.01)$ determined by one-way analysis of variance (ANOVA) followed by post hoc Tukey HSD analysis.

To unearth the functional genes enacting in the immune pathway regualated by the osa-miR1432/OsCaML2 module, the potential proteins interacting with OsCaML2 were screened from a yeast two-hybrid (Y2H) library, and two candidate proteins, OsHPGT3 and OsCSN6, were identified (Figure S6). OsCSN6 may be an iron deficiency responsive regulator to conquer Fe stress in rice [25]. The homology analysis and functional annotation suggest that OsHPGT3 may be involved in arabinogalactan metabolism of the rice cell wall, affecting the composition of the cell wall [26]. OsHPGT3 was chosen for further inspection. To determine whether the interaction between OsCaML2 and OsHPGT3 depends on the EF hand domain, the full-length OsCaML2 (1-213 aa) and two truncated variants, OsCaML2-N (containing EF hand domain at 146-174 aa) and OsCaML2-C (containing EF hand domain at 184-212 aa), were inserted into the pGBKT7 vector and co-transformed into yeast cells with pGADT7-OsHPGT3. The results showed that OsCaML2, OsCaML2-N and OsCaML2$C$ could interact with OsHPGT3 protein in yeast, indicating the interaction relies on the EF hand domain of OsCaML2 (Figure S7A-B). In addition, the bimolecular fluorescence complementation (BiFC) assay showed the interaction of OsCaML2 and OsHPGT3 also existed in Nicotiana benthamiana (Figure S7C). The above results suggest that OsHPGT3 may function as an osa-miR1432/OsCaML2 module-mediated defense pathway.

\section{Discussion}

Recent works have disclosed that miRNA/target modules serve as an indispensable participant in rice-pathogen interaction. Despite the fact that numerous miRNA/target modules have been identified by bioinformatic prediction or omics analysis, only two regulatory modules, osa-miR156/OsIPA1 [18] and osa-miR1876/NBS8R [19], were demon- 
strated to orchestrate rice immunity to Xoo utilizing the transgenic approach. In our study, overexpressing osa-miR1432 plants were shown to enhance rice resistance to Xoo invasion, while the endogenous blocking of osa-miR1432 avails Xoo multiplication in infected rice leaves. Consistently, the elevated accumulation of OsCaML2, the target of osa-miR1432, increases the susceptibility to Xoo invasion in rice. These results show that the osa-miR1432/OsCaML2 module is implicated in the immunity response to Xoo invasion and it is feasible to identify resistance-associated genes by exploiting the targets of functional miRNAs.

Previous work has illustrated the regulatory role of osa-miR1432 in developing rice seeds, which negatively regulates grain size via adjusting the grain filling rate through its target, rice Acyl-CoA thioesterase (OsACOT) [20]. Although the similar phenotype reappeared in the genetic materials of osa-miR1432 in this study, our attention was focused on the biological function of osa-miR1432 in rice-Xoo interaction, namely that osa-miR1432 operates as a positive immune response regulator. OsPBZ1, OsPR1 $a$ and OsPR1b, which were induced by salicylic acid (SA), jasmonic acid (JA) and ethylene (ET), respectively [27-29], in OE1432 plants, were significantly higher, relative to TP309 plants, and presented contrastive results in MIM1432 plants, implying that osa-miR1432-activated resistance may be involved in an SA-JA/ET-mediated pathway (Figures 2E and 3E). The Xoo resistance of OE1432 plants was associated with increased ROS accumulation and decreased growth rates of Xoo strain PXO86 during the period of rice-Xoo interaction (Figure 2D,F). In contrast, disturbing osa-miR1432 resulted in compromised resistance to Xoo invasion, which was attributable to less ROS accumulation and raised growth rates of Xoo strain PXO86 (Figure 3D,F).

The Xoo resistance endowed by overexpressing osa-miR1432 counts on the function inhibition of targets. Three potential regulatory modules, osa-miR1432/OsCaML2, osamiR1432/OsCAS and osa-miR1432/OsZIP6, were detected in our previous report [15]; however, the computational prediction of targets and expression assays of multiple timepoints between three candidate regulatory modules intensively sustained the notion that OsCaML2 may serve as a target participating in osa-miR1432-mediated rice resistance to Xoo. Meanwhile, though evolutionary analysis and bioinformatic prediction cannot separate LOC_Os03g59790 from OsCaML2 to function as a downstream actor, OsCaML2 could withstand the stringent filtration (category $\leq 2$ and raw reads $\geq 10$ in at least one library) of degradome data. On this basis, the GFP-based reporter assays point to the fact that OsCaML2 is the immune-responsive target of osa-miR1432 in rice-Xoo interaction. However, whether other potential targets are also implicated in osa-miR1432-mediated rice immunity to Xoo awaits answer in the future.

Calmodulin-like proteins (CMLs), containing EF hand motifs, were predicted to bind $\mathrm{Ca}^{2+}$ ions and were classified as calcium sensors [30,31]. The physiological relevance data of CMLs supported the idea that they may be involved in plant immune responses to pathogen invasion [32-34], and Soybean CMLs (SCaM-4/-5) and Arabidopsis CMLs (CML24, CML41, CML43, CML8, CML9) were confirmed to participate in the immune response of Pseudomonas syringae [35-41]. In our study, regarding the role of OsCaML2, a member of the rice CML family, rice immunity response conformed to the anticipation of targets for osa-miR1432 in rice-Xoo interactions. Consistent with the positive immune regulator of osa-miR1432, the overexpression of OsCaML2 exhibited a subdued resistance to Xoo attacks with the alteration of defense marker gene expressions, ROS levels and bacterial growth (Figure 5). Prompted by the role of the osa-miR1432/OsCaML2 module in Xoo infection, the participants acting in this immune pathway were surveyed, and OsHPGT3 and OsCSN6 were identified using Y2H technology (Figure S6). Depending on the homology analysis and functional annotation, OsHPGT3 involved in arabinogalactan metabolism of the rice cell wall was the top choice to investigate the defense pathway of the osa-miR1432/OsCaML2 module [26]. The possible interaction between OsCaML2 and OsHPGT3 revealed by $\mathrm{Y} 2 \mathrm{H}$ and BiFC assays suggests that it relies on the EF hand domain of OsCaML2 (Figure S7A-C). A working hypothesis was that the osa-miR1432-directed 
OsCaML2 repression may thicken secondary cell walls and increase lignin content by affecting the galactosyl transferase activity of OsHPGT3 protein, improving resistance to $\mathrm{Xoo}$ in rice.

\section{Materials and Methods}

\subsection{Plant Materials and Growth Condition}

Rice (Oryza sativa L.) plants were cultivated in the experimental field at Changping, Beijing during the natural growing season. The Xoo-sensitive genotypes, IR24 and its insensitive derivative, IRBB5, served to appraise the pathogenicity of Xoo strain PXO86. All rice transgenic lines were generated in the background of Japonica rice cultivar TP309 and used for experiment in the $\mathrm{T}_{2}$ generation.

\subsection{Pathogen Inoculation and Disease Assays}

Xoo strains PXO86, PXO112, PXO99 and PXO339, preserved in a $-80{ }^{\circ} \mathrm{C}$ freezer, were streaked on PSA (10 g/L tryptone, $10 \mathrm{~g} / \mathrm{L}$ sucrose, $1 \mathrm{~g} / \mathrm{L}$ sodium-glutamate and $10 \mathrm{~g} / \mathrm{L}$ agar, $\mathrm{pH}=7.0)$ plates with cephalexin $(15 \mathrm{mg} / \mathrm{L})$ and incubated at $28^{\circ} \mathrm{C}$. After $3 \mathrm{~d}$, the cells harvested from the plates were resuspended in sterilized distilled water $\left(\mathrm{OD}_{600}=0.5\right)$. Rice leaves were clipped using scissors with Xoo suspension or water at the tillering stage [42], and the lesion lengths of 15 individual plants with three repeats were measured $20 \mathrm{~d}$ after inoculation for each strain.

For bacterial growth analysis, 3 infected leaves from independent lines were adequately ground utilizing a mortar and pestle. Then, the milled leaf was suspended in $10 \mathrm{~mL}$ of bacteria-free water, and the diluted mixture was transferred to PSA plates containing $15 \mathrm{mg} / \mathrm{L}$ of cephalexin. Finally, the cell colonies were counted from the plates incubated at $28^{\circ} \mathrm{C}$ for $3 \mathrm{~d}$.

\subsection{Vector Construction and Rice Transformation}

For functional overexpression, osa-miR1432 precursors and OsCaML2 were amplified from TP309 genomic sequence and full-length complementary DNA (cDNA), respectively. The fragments were cloned into the plant binary vector, UBI-pCAMBIA1300, and then transformed into TP309 calli via Agrobacterium strain EHA105-mediated transformation. To create a target mimicry construct for silencing osa-miR1432 expression, we inserted the artificial target mimic of osa-miR1432 into the IPS1 gene [23]. Then, the mutated fragments were cloned into the binary vector, pCAMBIA1300, to generate MIM1432 construct, which was introduced into TP309 calli through Agrobacterium strain EHA105-mediated transformation. All primers for vector construction are listed in Table S1.

\subsection{Quantitative Reverse Transcription-PCR Assay}

For gene expression analysis, total RNA was extracted from leaves at different collected time points with or without Xoo treatment using TRIzol reagent (Invitrogen, Waltham, MA, USA) and reverse transcribed to cDNA using the ReverTra Ace 1 qPCR RT Master Mix with gDNA Remover (TOYOBO, Osaka, Japan). To monitor osa-miR1432, the miRNAs were extracted from the aforementioned samples using an miRNA Isolation Kit (OMEGA, Atlanta, GA, USA), and the specific stem-loop RT primer for osa-miR1432 was applied to the reverse transcription protocol [43]. Quantitative reverse transcription-PCR (qRT-PCR) was performed using indicated primers and TransStart Green qRT-PCR Super Mix (TransGen, Beijing, China). SnRNA U6 and OsACTIN genes separately served as the internal reference to data normalization for miRNAs and mRNAs, and the relative expression levels of miRNAs and target genes were determined using a one-way ANOVA followed by post hoc Tukey HSD analysis with three biological replicates and at least three technical repeats. Specific primers for quantitative real-time PCR are listed in Supplementary Table S1. 


\subsection{Transient Expression Analysis in Nicotiana Benthamiana}

Transient co-expression assays were carried out by injecting the Agrobacterium strain EHA105 mixtures, which harbored the respective expression constructs, 35S::miR1432 (pCAMBIA1300), 35S::OsCaML2 (pCAMBIA1300-221-GFP) and 35S::MIM1432 (pCAMBIA1300), into leaves of 4-week-old N. benthamiana plants. In brief, Agrobacterium strain EHA105 was shaken at $28{ }^{\circ} \mathrm{C}$ overnight in LB liquid media containing kanamycin $(50 \mathrm{mg} / \mathrm{mL})$ and rifampicin $(50 \mathrm{mg} / \mathrm{mL})$ with $250 \mathrm{rpm}$. The cells were collected at $8000 \mathrm{rpm}$ for $3 \mathrm{~min}$ and resuspended in an MMA buffer (10 mM MES, $10 \mathrm{mM} \mathrm{MgCl}_{2}, 200 \mu \mathrm{M}$ acetosyringone, $\mathrm{pH}=5.6$ ) until $\mathrm{OD}_{600}=1.0$. After rest at temperature for $2-3 \mathrm{~h}$, the Agrobacterium strain EHA105 containing the destination constructs were infiltrated into leaves of $N$. benthamiana for the transient expression assay. GFP accumulation was detected at $48 \mathrm{~h}$ post infiltration (hpi) for imaging using ZEISS LSM 710 NLO (Carl Zeiss, Oberkochen, Germany)

\subsection{Oxidative Burst Assays}

DAB and NBT staining were conducted for the determination of $\mathrm{H}_{2} \mathrm{O}_{2}$ and $\mathrm{O}_{2}{ }^{-}$with modified protocol [44]. Briefly, 15 excised rice leaf sections from 5 independent lines were submerged in the staining solution $(10 \mathrm{mM}$ Tris $-\mathrm{HCl}(\mathrm{pH}=6.5)$ containing $1 \mathrm{mg} / \mathrm{mL}$ of 1 DAB (Sigma, St. Louis, MO, USA) or $10 \mathrm{mM}$ sodium citrate $(\mathrm{pH}=6.0)$ containing $0.05 \%$ NBT (Sigma, St. Louis, MO, USA) and then incubated at $37^{\circ} \mathrm{C}$ in the dark for $12 \mathrm{~h}$. The chlorophyll of leaves was washed out with bleaching solution (ethanol:acetic acid:glycerol $=3: 1: 1)$ in a boiling water bath $\left(90-95^{\circ} \mathrm{C}\right)$ until the leaves became clear. Finally, the stained leaves were photographed using an HP LJ M1530 scanner (Hewlett-Packard, Palo Alto, CA, USA).

\section{7. $\mathrm{Y} 2 \mathrm{H}$ Assays}

$\mathrm{Y} 2 \mathrm{H}$ assays were performed in consonance with the Matchmaker Gold Y2H System user's manual (Clontech, Mountain View, CA, USA). The full coding sequences, truncated $\mathrm{N}$ and $\mathrm{C}$ terminal coding region of $\mathrm{OsCaML2}$, were introduced into the pGBKT7 vector. The bait plasmid pGBKT7-OsCaML2 was co-transformed with rice cDNA library for screening interacting proteins on SD/-Leu-Trp-selected plates, and different dilutions $\left(10^{-1}, 10^{-2}\right.$ and $10^{-3}$ ) of positive clones were transferred to $\mathrm{SD} /$-Trp-Leu-His-Ade medium with $\beta$ galactosidase $(X-\alpha-\mathrm{Gal})$ to confirm the interaction. After incubation at $30^{\circ} \mathrm{C}$ for 3 or $4 \mathrm{~d}$, yeast growth was assessed. By the same method, the coding sequence of OsHGPT3 was cloned into pGADT7 for specific interaction of OsCaML2 and OsHGPT3.

\subsection{BiFC Assay}

The full-length coding regions of OsCaML2 and OsHGPT3 were amplified and cloned into the pVYNE and pVYCE vectors, respectively. The recombinant vectors were cotransformed into 4-week-old N. benthamiana leaves via Agrobacterium strain EHA105mediated transient expression. Briefly, the bacteria cells were collected and resuspended in infection solution (10 mM MES, $10 \mathrm{mM} \mathrm{MgCl}_{2}$ and $200 \mu \mathrm{M}$ of acetosyringone, $\mathrm{pH}=5.6$ ). The prepared suspensions were infiltrated into 4 -week-old $N$. benthamiana leaves, and the infiltrated $N$. benthamiana plants were grown for $2 \mathrm{~d}$. Fluorescent eYFP signals were detected and photographed using ZEISS LSM 710 NLO (Carl Zeiss, Oberkochen, Germany.excitation $514 \mathrm{~nm}$, emission 525-565 nm).

\section{Conclusions}

In conclusion, our study demonstrates the regulatory roles of the osa-miR1432/ OsCaML2 module in the resistance of rice against Xoo and displays new evidence for capturing minor resistance-associated genes by anchoring the targets of miRNAs, which may offer direction for the breeding or genetic engineering of Xoo-resistant rice plants.

Supplementary Materials: The following are available online at https:/ / www.mdpi.com/article/10 $.3390 /$ ijms222111367/s1. 
Author Contributions: Conceptualization, Y.J. and C.L.; methodology, Y.J.; software, Y.J. and Q.L.; validation, Y.J.; formal analysis, C.L., Y.J. and Q.L.; investigation, Y.J., Q.L., Y.L. and C.L.; resources, W.Z. and G.J.; data curation, Y.J.; writing-original draft preparation, Y.J. and Q.L.; writing-review and editing, Y.J. and Q.L.; visualization, Y.J.; supervision, Y.J., Q.L., C.L., W.Z. and G.J.; project administration, W.Z.; funding acquisition, C.L. All authors have read and agreed to the published version of the manuscript.

Funding: This work was supported by the National Natural Science Foundation of China (31900383) and project funded by China Postdoctoral Science Foundation (2019M660853).

Institutional Review Board Statement: Not applicable.

Informed Consent Statement: Not applicable.

Conflicts of Interest: The authors declare no conflict of interest.

$\begin{array}{ll}\text { Abbreviations } \\ \text { BiFC } & \text { Bimolecular fluorescence complementation } \\ \text { DAB } & \text { 3,3'-diaminobenzidine } \\ \text { CMLs } & \text { Calmodulin-like proteins } \\ \text { dpi } & \text { Days post inoculation } \\ \text { ET } & \text { Ethylene } \\ \text { GA } & \text { Gibberellin } \\ \text { GFP } & \text { Green fluorescent protein } \\ \text { hpi } & \text { Hours post infection } \\ \text { JA } & \text { Jasmonic acid } \\ \text { miRNAs } & \text { MicroRNAs } \\ \text { RT-qPCR } & \text { Reverse transcription quantitative PCR } \\ \text { OsCaML2 } & \text { LOC_Os03g59770 } \\ \text { OsCAS } & \text { LOC_Os04g08350 } \\ \text { OsCSN6 } & \text { LOC_Os08g39070 } \\ \text { OE } & \text { Overexpressing } \\ \text { OsHPGT3 } & \text { LOC_Os06g07905 } \\ \text { OsZIP6 } & \text { LOC_Os05g07210 } \\ \text { OsPBZ1 } & \text { Oryza sativa pathogenesis-related protein 10a } \\ \text { OsPR1a } & \text { Oryza sativa pathogenesis-related protein 1a } \\ \text { OsPR1b } & \text { Oryza sativa pathogenesis-related protein 1b } \\ \text { Rice } & \text { Oryza sativa L. } \\ \text { ROS } & \text { Reactive oxygen species } \\ \text { SA } & \text { Salicylic acid } \\ \text { TP309 } & \text { Taipei 309 } \\ \text { Xoo } & \text { Xanthomonas oryzae pv. oryzae } \\ \text { Y2H } & \text { Yeast two-hybrid } \\ & \end{array}$

\section{References}

1. Song, X.W.; Li, Y.; Cao, X.F.; Qi, Y.J. MicroRNAs and their regulatory roles in plant-environment interactions. Annu. Rev. Plant Biol. 2019, 70, 489-525. [CrossRef] [PubMed]

2. Lee, B.M.; Park, Y.J.; Park, D.S.; Kang, H.W.; Kim, J.G.; Song, E.S.; Park, I.C.; Yoon, U.H.; Hahn, J.H.; Koo, B.S.; et al. The genome sequence of Xanthomonas oryzae pathovar oryzae KACC10331, the bacterial blight pathogen of rice. Nucleic Acids Res. 2005, 33, 577-586. [CrossRef]

3. Liu, W.; Liu, J.; Triplett, L.; Leach, J.E.; Wang, G.L. Novel insights into rice innate immunity against bacterial and fungal pathogens. Annu. Rev. Phytopathol. 2014, 52, 213-241. [CrossRef] [PubMed]

4. Mansfield, J.; Genin, S.; Magori, S.; Citovsky, V.; Sriariyanum, M.; Ronald, P.; Dow, M.; Verdier, V.; Beer, S.V.; Machado, M.A.; et al. Top 10 plant pathogenic bacteria in molecular plant pathology. Mol. Plant Pathol. 2012, 13, 614-629. [CrossRef] [PubMed]

5. Jambhulkar, P.P.; Sharma, P. Developent of bioformulation and delivery system of Pseudomonas fluorescens against bacterial leaf blight of rice (Xanthomonas oryzae pv. oryzae). J. Environ. Biol. 2014, 35, 843-849. [PubMed]

6. Poulin, L.; Grygiel, P.; Magne, M.; Gagnevin, L.; Rodriguez-R, L.M.; Serna, N.F.; Zhao, S.; Rafii, M.E.; Dao, S.; Tekete, C.; et al. New multilocus variable-number tandem-repeat analysis tool for surveillance and local epidemiology of bacterial leaf blight and bacterial leaf streak of rice caused by Xanthomonas oryzae. Appl. Environ. Microb. 2015, 81, 688-698. [CrossRef] [PubMed] 
7. Fahad, S.; Nie, L.X.; Khan, F.A.; Chen, Y.T.; Hussain, S.; Wu, C.; Xiong, D.L.; Jing, W.; Saud, S.; Khan, F.A.; et al. Disease resistance in rice and the role of molecular breeding in protecting rice crops against diseases. Biotechnol. Lett. 2014, 36, 1407-1420. [CrossRef]

8. White, F.F.; Yang, B. Host and pathogen factors controlling the rice-Xanthomonas oryzae interaction. Plant Physiol. 2009, 150, 1677-1686. [CrossRef]

9. Hop, D.V.; Hoa, P.T.; Quang, N.D.; Ton, P.H.; Ha, T.H.; Huang, N.V.; Van, N.T.; Hai, T.V.; Quy, N.T.; Dao, N.T.; et al. Biological control of Xanthomonas oryzae pv. oryzae causing rice bacterial blight disease by Streptomyces toxytricini VN08-A-12, isolated from soil and leaf-litter samples in Vietnam. Biocontrol. Sci. 2014, 19, 103-111. [CrossRef]

10. Dilla-Ermita, C.J.; Tandayu, E.; Juanillas, V.M.; Detras, J.; Lozada, D.N.; Dwiyanti, M.S.; Cruz, C.V.; Mbanjo, E.G.; Ardales, E.; Diaz, M.G.; et al. Genome-wide association analysis tracks bacterial leaf blight resistance loci in rice diverse germplasm. Rice 2017, 10, 8. [CrossRef]

11. Kim, S.M.; Reinke, R.F. A novel resistance gene for bacterial blight in rice, Xa43(t) identified by GWAS, confirmed by QTL mapping using a bi-parental population. PLoS ONE 2019, 14, e0211775. [CrossRef]

12. Oliva, R.; Ji, C.H.; Atienza-Grande, G.; Huguet-Tapia, J.C.; Perez-Quintero, A.; Li, T.; Li, J.S.; Li, C.H.; Nguyen, H.; Liu, B.; et al. Broad-spectrum resistance to bacterial blight in rice using genome editing. Nat. Biotechnol. 2019, 37, 1344-1350. [CrossRef]

13. Xu, Z.Y.; Xu, X.M.; Gong, Q.; Li, Z.Y.; Li, Y.; Wang, S.; Yang, Y.Y.; Ma, W.X.; Liu, L.Y.; Zhu, B.; et al. Engineering broad-spectrum bacterial blight resistance by simultaneously disrupting variable TALE-binding elements of multiple susceptibility genes in rice. Mol. Plant 2019, 112, 1434-1446. [CrossRef] [PubMed]

14. Hong, H.M.; Liu, Y.Y.; Zhang, H.T.; Xiao, J.H.; Li, X.H.; Wang, S.P. Small RNAs and gene network in a durable disease resistance gene-mediated defense responses in rice. PLOS ONE 2015, 10, e0137360.

15. Jia, Y.F.; Li, C.R.; Li, Q.L.; Liu, P.C.; Liu, D.F.; Liu, Z.Z.; Wang, Y.Y.; Jiang, G.H.; Zhai, W.X. Characteristic dissection of Xanthomonas oryzae pv. oryzae responsive microRNAs in rice. Int. J. Mol. Sci. 2020, 21, 785. [CrossRef] [PubMed]

16. Zhang, S.; Mei, J.; Wang, T.; Wang, C.C.; Zhang, W.L.; Yang, L. Identification and expression analysis of OsmiR1861k in rice leaves in response to Xanthomonas oryzae pv. oryzae. J. Gen. Plant Pathol. 2015, 81, 108-117. [CrossRef]

17. Yu, C.; Chen, Y.T.; Cao, Y.Q.; Chem, H.M.; Wang, J.C.; Bi, Y.M.; Tian, F.; Yang, F.H.; Rothstein, S.J.; Zhou, X.P.; et al. Overexpression of miR169o, an overlapping microRNA in response to both nitrogen limitation and bacterial infection, promotes nitrogen use efficiency and susceptibility to bacterial blight in rice. Plant. Cell Physiol. 2018, 59, 1234-1247. [CrossRef] [PubMed]

18. Liu, M.M.; Shi, Z.Y.; Zhang, X.H.; Wang, M.X.; Zhang, L.; Zheng, K.Z.; Liu, J.Y.; Hu, X.M.; Di, C.R.; Qian, Q.; et al. Inducible overexpression of Ideal Plant Architecture1 improves both yield and disease resistance in rice. Nat. Plants 2019, 5, 389-400. [CrossRef]

19. Jiang, G.H.; Liu, D.F.; Yin, D.D.; Zhou, Z.Z.; Shi, Y.; Li, C.R.; Zhu, L.H.; Zhai, W.X. A rice NBS-ARC gene conferring quantitative resistance to bacterial blight is regulated by a pathogen effector-inducible miRNA. Mol. Plant 2020, 20, 30310-30325. [CrossRef]

20. Zhao, Y.F.; Peng, T.; Sun, H.Z.; Teotia, S.; Wen, H.L.; Du, Y.X.; Zhang, J.; Li, J.Z.; Tang, G.L.; Xue, H.W.; et al. miR1432-OsACOT (Acyl-CoA thioesterase) module determines grain yield via enhancing grain filling rate in rice. Plant Biotechnol. J. 2019, 17, 712-723. [CrossRef]

21. Huang, L.F.; Lin, K.H.; He, S.L.; Chen, J.L.; Jiang, J.Z.; Chen, B.H.; Hou, Y.S.; Chen, R.S.; Hong, C.Y.; Ho, S.L. Multiple patterns of regulation and overexpression of a ribonuclease-like pathogenesis-related protein gene, OsPR10a, conferring disease resistance in rice and Arabidopsis. PLoS ONE 2016, 11, e0156414. [CrossRef]

22. Mitsuhara, I.; Iwai, T.; Seo, S.; Yanagawa, Y.; Kawahigasi, H.; Hirose, S.; Ohkawa, Y.; Ohashi, Y. Characteristic expression of twelve rice PR1 family genes in response to pathogen infection, wounding, and defense-related signal compounds (121/180). Mol. Genet. Genomics 2008, 279, 415-427. [CrossRef]

23. Franco-Zorrilla, J.M.; Valli, A.; Todesco, M.; Mateos, I.; Puga, M.I.; Rubio-Somoza, I.; Leyva, A.; Weigel, D.; Garcia, J.A.; Paz-Ares, J. Target mimicry provides a new mechanism for regulation of microRNA activity. Nat. Genet. 2007, 39, 1033-1037. [CrossRef]

24. Todesco, M.; Rubio-Somoza, I.; Paz-Ares, J.; Weigel, D. A collection of target mimics for comprehensive analysis of microRNA function in Arabidopsis thaliana. PLoS Genet. 2010, 6, e1001031. [CrossRef] [PubMed]

25. Tan, S.; Liu, F.; Pan, X.X.; Zang, Y.P.; Jin, F.; Zu, W.X.; Qi, X.T.; Xiao, W.; Yin, L.P. CSN6, a subunit of the COP9 signalosome, is involved in early response to iron deficiency in Oryza sativa. Sci. Rep. 2016, 6, 25485. [CrossRef]

26. Showalter, A.M.; Basu, D. Glycosylation of arabinogalactan-proteins essential for development in Arabidopsis. Commun. Integ. Biol. 2016, 9, e1177687. [CrossRef] [PubMed]

27. Hwang, S.H.; Lee, I.A.; Yie, S.W.; Hwang, D.J. Identification of an OsPR10a promoter region responsive to salicylic acid. Planta 2008, 227, 1141-1150. [CrossRef]

28. Rakwal, R.; Agrawal, G.K.; Yonekura, M. Light-dependent induction of OsPR10 in rice (Oryza sativa L.) seedlings by the global stress signaling molecule jasmonic acid and protein phosphatase 2A inhibitors. Plant Sci. 2001, 161, 469-479. [CrossRef]

29. Nahar, K.; Kyndt, T.; Vleesschauwer, D.D.; Höfte, M.; Gheysen, G. The jasmonate pathway is a key player in systemically induced defense against root knot nematodes in rice. Plant Physiol. 2011, 157, 305-316. [CrossRef]

30. McCormack, E.; Tsai, Y.C.; Braam, J. Handling calcium signaling: Arabidopsis CaMs and CMLs. Trends Plant Sci. 2005, 10, 383-389. [CrossRef]

31. Zhu, X.; Dunand, C.; Snedden, W.; Galaud, J.P. CaM and CML emergence in the green lineage. Trends Plant Sci. 2015, 20, 483-489. [CrossRef] 
32. Ranty, B.; Aldon, D.; Cotelle, V.; Galaud, J.P.; Thuleau, P.; Mazars, C. Calcium sensors as key hubs in plant responses to biotic and abiotic stresses. Front. Plant Sci. 2016, 7, 327-333. [CrossRef]

33. Bender, K.W.; Snedden, W.A. Calmodulin-related proteins step out from the shadow of their namesake. Plant Physiol. 2013, 163, 486-495. [CrossRef]

34. Cheval, C.; Aldon, D.; Galaud, J.P.; Ranty, B. Calcium/calmodulin-mediated regulation of plant immunity. Biochim. Biophys. Acta 2013, 1833, 1766-1771. [CrossRef]

35. Park, C.Y.; Heo, W.D.; Yoo, J.H.; Lee, J.H.; Kim, M.C.; Chun, H.J.; Moon, B.C.; Kim, I.H.; Park, H.C.; Choi, M.S.; et al. Pathogenesisrelated gene expression by specific calmodulin isoforms is dependent on nim1, a key regulator of systemic acquired resistance. Mol. Cells 2004, 18, 207-213. [PubMed]

36. Chiasson, D.; Ekengren, S.K.; Martin, G.B.; Dobney, S.L.; Snedden, W.A. Calmodulin-like proteins from Arabidopsis and tomato are involved in host defense against Pseudomonas syringae pv. Tomato. Plant Mol. Biol. 2005, 58, 887-897. [CrossRef] [PubMed]

37. Ma, W.; Smigel, A.; Tsai, Y.C.; Braam, J.; Berkowitz, G.A. Innate immunity signaling: Cytosolic Ca ${ }^{2+}$ elevation is linked to downstream nitric oxide generation through the action of calmodulin or a calmodulin-like protein. Plant Physiol. 2008, 148, 818-828. [CrossRef] [PubMed]

38. Leba, L.J.; Cheval, C.; Ortiz-Martin, I.; Ranty, B.; Beuzon, C.R.; Galaud, J.P.; Aldon, D. CML9, an Arabidopsis calmodulin-like protein, contributes to plant innate immunity through a flagellin-dependent signalling pathway. Plant J. 2012, 71, 976-989. [CrossRef]

39. Zhu, X.; Robe, E.; Jomat, L.; Aldon, D.; Mazars, C.; Galaud, J.P. CML8, an Arabidopsis calmodulin-like protein, plays a role in pseudomonas syringae plant immunity. Plant Cell Physiol. 2017, 58, 307-319.

40. Zhu, X.; Perez, M.; Aldon, D.; Galaud, J.P. Respective contribution of CML8 and CML9, two Arabidopsis calmodulin-like proteins, to plant stress responses. Plant Signal Behav. 2017, 12, e1322246. [CrossRef]

41. Xu, B.; Cheval, C.; Laohavisit, A.; Hocking, B.; Chiasson, D.; Olsson, T.S.G.; Shirasu, K.; Faulkner, C.; Gilliham, M. A calmodulinlike protein regulates plasmodesmal closure during bacterial immune responses. New Phytol. 2017, 215, 77-84. [CrossRef] [PubMed]

42. Chen, H.L.; Wang, S.P.; Zhang, Q.F. New gene for bacterial blight resistance in rice located on chromosome 12 identified from minghui 63, an elite restorer line. Phytopathology 2002, 92, 750-754. [CrossRef] [PubMed]

43. Varkonyi-Gasic, E.; Wu, R.; Wood, M.; Walton, E.F.; Hellens, R.P. Protocol: A highly sensitive RT-PCR method for detection and quantification of microRNAs. Plant Methods 2007, 3, 12-21. [CrossRef] [PubMed]

44. Xia, X.J.; Wang, Y.J.; Zhou, Y.H.; Tao, Y.; Mao, W.H.; Shi, K.; Asami, T.; Chen, Z.X.; Yu, J.Q. Reactive oxygen species are involved in brassinosteroid-induced stress tolerance in cucumber. Plant Physiol. 2009, 150, 801-814. [CrossRef] 